PAPER

Fabrication and characterization of graphene/AIGaN/GaN ultraviolet Schottky photodetector

To cite this article: M Kumar et al 2016 J. Phys. D: Appl. Phys. 49275105

View the article online for updates and enhancements.
Related content

$$
\begin{aligned}
& \text { - Demonstration of a large-area AIGaN/GaN } \\
& \frac{\text { Schottky barrier photodetector on Si with }}{\text { high detection limit }} \\
& \text { Manoj Kumar, Chang Yong Lee, Hiroto } \\
& \text { Sekiguchi et al. } \\
& \text { - A metal-semiconductor-metal detector } \\
& \frac{\text { based on ZnO nanowires grown on a }}{\text { graphene laver }} \\
& \text { Qiang Xu, Qijin Cheng, Jinxiang Zhong et } \\
& \text { al. } \\
& \text { - Large bandgap reduced graphene oxide } \\
& \frac{\text { (rGO) based n-p+ heterojunction }}{\text { photodetector with improved NIR }} \\
& \text { performance } \\
& \text { Manjri Singh, Gaurav Kumar, Nisha } \\
& \text { Prakash et al. }
\end{aligned}
$$

Recent citations

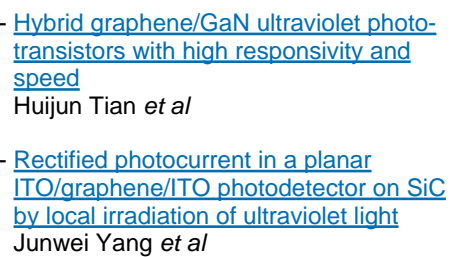




\title{
Fabrication and characterization of graphene/AIGaN/GaN ultraviolet Schottky photodetector
}

\author{
M Kumar ${ }^{1}$, H Jeong ${ }^{1}$, K Polat $^{2}$, A K Okyay ${ }^{2}$ and D Lee ${ }^{1}$ \\ ${ }^{1}$ School of Mechanical Engineering, Konkuk University, 120 Neungdong-ro, Gwangjin-gu, \\ Seoul 143-701, Korea \\ ${ }^{2}$ UNAM-National Nanotechnology Research Center and Institute of Materials Science and \\ Nanotechnology, Bilkent University, 06800 Ankara, Turkey \\ E-mail: djlee@konkuk.ac.kr
}

Received 20 January 2016, revised 18 March 2016

Accepted for publication 29 March 2016

Published 7 June 2016

\begin{abstract}
We report on the fabrication and characterization of a Schottky ultraviolet graphene/AlGaN/ GaN photodetector (PD). The fabricated device clearly exhibits rectification behaviour, indicating that the Schottky barrier is formed between the $\mathrm{AlGaN}$ and the mechanically transferred graphene. The Schottky parameters are evaluated using an equivalent circuit with two diodes connected back-to-back in series. The PD shows a low dark current of $4.77 \times 10^{-12} \mathrm{~A}$ at a bias voltage of $-2.5 \mathrm{~V}$. The room temperature current-voltage $(I-V)$ measurements of the graphene/AlGaN/GaN Schottky PD exhibit a large photo-to-dark contrast ratio of more than four orders of magnitude. Furthermore, the device shows peak responsivity at a wavelength of $350 \mathrm{~nm}$, corresponding to $\mathrm{GaN}$ band edge and a small hump at $300 \mathrm{~nm}$ associated to the AlGaN band edge. In addition, we examine the behaviour of Schottky PDs with responsivities of 0.56 and $0.079 \mathrm{~A} \mathrm{~W}^{-1}$ at 300 and $350 \mathrm{~nm}$, respectively, at room temperature.
\end{abstract}

Keywords: photodetector, grapheme, Schottky contact, AlGaN, GaN

(Some figures may appear in colour only in the online journal)

\section{Introduction}

As a result of recent advancements in material growth technology, high-quality $\mathrm{AlGaN} / \mathrm{GaN}$ templates have gained significant attention for the fabrication of high-temperature and high-power devices operating in the ultraviolet (UV) spectral region. For instance, AlGaN/GaN-based UV photodetectors (PDs) have been investigated for many years owing to their extensive applications in missile detection, secure space-to-space communication, and atmospheric monitoring [1-3]. Various types of devices have been studied, including those that employ the typical p-i-n structure, the inverted $\mathrm{p}-\mathrm{i}-\mathrm{n}$ structure, the Schottky barrier photodiode, and the inverted Schottky photodiode [4-7]. At the same time, graphene has recently attracted a strong interest in transparent and conducting electrodes because of its impressive electrical conductivity, high optical transparency, mechanical flexibility, two-dimensional (2D) structure, short carrier lifetime, and mechanical flexibility [8-10]. Accordingly, graphene layers can play vital roles as carrier transport layers and as electrodes in ultrafast PDs.

Recently, several studies have shown that graphene can be used to successfully form a Schottky electrode with conventional semiconductors such as $\mathrm{GaAs}, \mathrm{SiC}, \mathrm{GaN}$, and $\mathrm{Si}$ [11-14]. Graphene/nanowire semiconductor-based devices demonstrated extensive applications in the areas of solar cells, UV PDs, light-emitting devices, and gas-sensing devices [15-18]. Nevertheless, metal electrodes have poor transparencies and can dramatically influence the absorption efficiency of UV sensors. Thermally and mechanically stable graphene and multi-layer graphene contacts are therefore potential candidates for replacing conventional metal contacts, which 


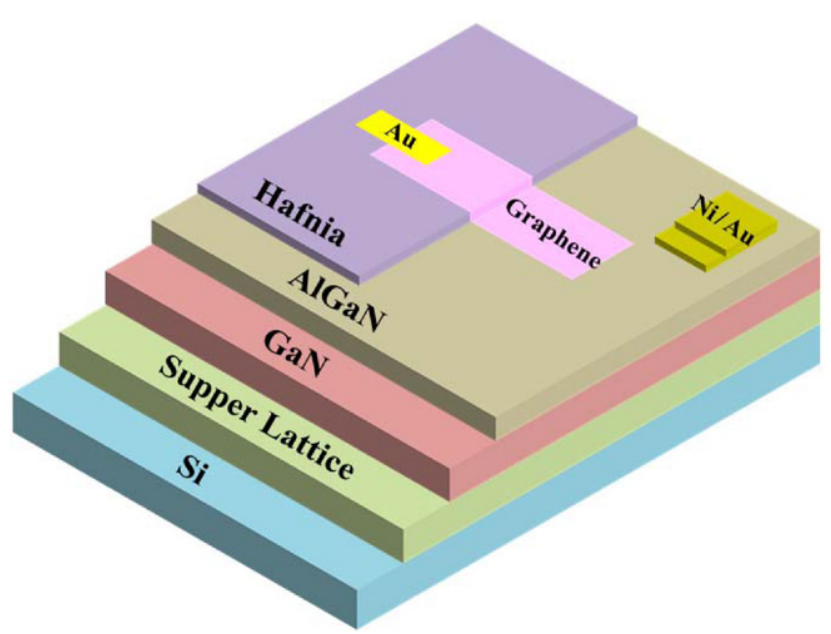

Figure 1. Schematic diagram of the fabricated graphene/AlGaN/ GaN Schottky photodetector.

degrade easily at high temperatures by diffusing into the semiconductor and irreversibly forming undesirable Ohmic contacts. Moreover, graphene-based PDs exhibit high speeds and high photoresponsivities through a broad spectral wavelength range, as well as ultrafast response times. Nevertheless, very few studies concerning heterojunctions between graphene and the well-known III-V semiconductor GaN have been performed [19].

In this study, performance of a graphene-based Schottky PD on an $\mathrm{AlGaN} / \mathrm{GaN}$ template was investigated. The chemical vapour deposition (CVD)-grown graphene was transferred to the $\mathrm{AlGaN} / \mathrm{GaN}$ template by a wet transfer method resulting in continuous coverage over a large area. In this manner, the entire contact area between the graphene and $\mathrm{AlGaN} / \mathrm{GaN}$ was able to function as the effective photoresponsive area. The fabricated device exhibits a low dark current of $4.77 \times 10^{-12} \mathrm{~A}$ at a bias of $-2.5 \mathrm{~V}$.

\section{Experimental details}

A schematic diagram of the fabricated graphene/AlGaN/ GaN Schottky PD is shown in figure 1. The $\mathrm{Al}_{0.25} \mathrm{GaN}_{0.75} /$ GaN grown by metal organic CVD on a $\mathrm{Si}$ wafer used in this study was commercially available. The $\mathrm{Al}_{0.25} \mathrm{GaN}_{0.75} / \mathrm{GaN}$ on the $\mathrm{Si}$ was cleaned using acetone and isopropyl alcohol, and an unintentionally grown oxide layer was etched by buffered hydrofluoric acid. Hafnia $\left(\mathrm{HfO}_{2}\right)$ with a thickness of $40 \mathrm{~nm}$ was deposited on half of the $\mathrm{AlGaN} / \mathrm{GaN}$ surface with shadow mask using an atomic layer deposition system (Ultratech/Cambridge Nanotech Savannah 100). The tetrakis (Dimethylamido) hafnium and $\mathrm{H}_{2} \mathrm{O}$ were used as precursors.

High-quality graphene was synthesized by $\mathrm{CVD}$ on $\mathrm{Cu}$ foil. Prior to the deposition of graphene, the $\mathrm{Cu}$ foil was cleaned with acetone and isopropyl alcohol, and was then rinsed with deionized water. The base pressure of the chamber was maintained at $1.5 \mathrm{~m}$ Torr and the temperature was maintained at $950{ }^{\circ} \mathrm{C}$. After growing the graphene on the $\mathrm{Cu}$ foil, it was spin-coated with polymethylmethacrylate (PMMA). The PMMA-coated $\mathrm{Cu}$ foil was soaked in a diluted nitric acid

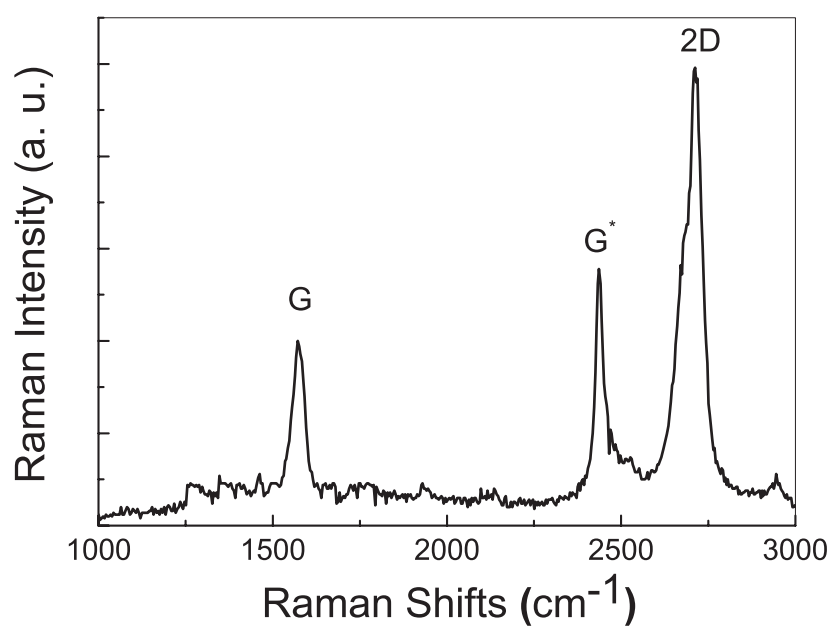

Figure 2. Raman spectrum of graphene layer transferred onto the AlGaN/GaN substrate.

solution in order to remove the graphene from the bottom side, and the $\mathrm{Cu}$ foil was then dissolved in an ammonium persulphate solution. Subsequently, the PMMA/graphene was lifted from the solution and transferred into $\mathrm{H}_{2} \mathrm{O}$. The PMMA/ graphene was then transferred onto the substrate such that half of the PMMA/graphene covered the $\mathrm{Al}_{0.25} \mathrm{GaN}_{0.75} / \mathrm{GaN}$ layer directly, whereas the other half was placed on top of the $\mathrm{HfO}_{2}$ layer. After drying, the PMMA was removed by acetone.

The Au electrode was fabricated across the $\mathrm{HfO}_{2}$ and graphene portions; the other electrode $(\mathrm{Au} / \mathrm{Ni})$ was fabricated directly on the $\mathrm{Al}_{0.25} \mathrm{GaN}_{0.75} / \mathrm{GaN}$ using a magnetron sputtering technique. The $\mathrm{Au}(100 \mathrm{~nm}$ thick) was deposited at constant power of $75 \mathrm{~W}$, a gas flow of $50 \mathrm{sccm}$, and a pressure of 1 mTorr. High-purity Ar gas was used during the sputtering process. For the deposition of the $10 \mathrm{~nm}$ thick Ni layer, we used a power of $125 \mathrm{~W}$, a gas flow rate of $50 \mathrm{sccm}$, and a pressure of 20 mTorr. Metal deposition was carried out in a vacuum chamber evacuated to a pressure of $5.6 \times 10^{-6}$ Torr.

Current-voltage $(I-V)$ measurements were performed using a semiconductor parameter analyser (Keithley 4200) and a source meter (Keithley 2400). The spectral response was measured using a lock-in amplifier with an optical chopper and a monochromator in the wavelength range of 250-450 nm with a $150 \mathrm{~W}$ Xe arc lamp. The Xe lamp intensity was maintained $36 \mu \mathrm{W}$ during the measurement.

\section{Results}

Figure 2 shows the Raman spectrum of the transferred graphene, which reveals peaks at 1569,2435 and $2711 \mathrm{~cm}^{-1}$. The peaks located at 1569 and $2711 \mathrm{~cm}^{-1}$ can be attributed to the $\mathrm{G}$ and 2D modes of the graphene, respectively, which is consistent with previous studies [20,14]. The number of layers in the transferred graphene is associated with the intensity ratio of the $2 \mathrm{D}$ to $\mathrm{G}$ modes. The Raman intensity ratio of the $2 \mathrm{D}$ to $\mathrm{G}$ modes is found to be $1: 2.5$, indicating the monolayer nature of graphene [21]. The peak located at $2435 \mathrm{~nm}$ is associated to $G^{*}$ Raman band. The $G^{*}$ Raman band reveals the defect activated peak in the Raman Spectrum [22]. However, the D 


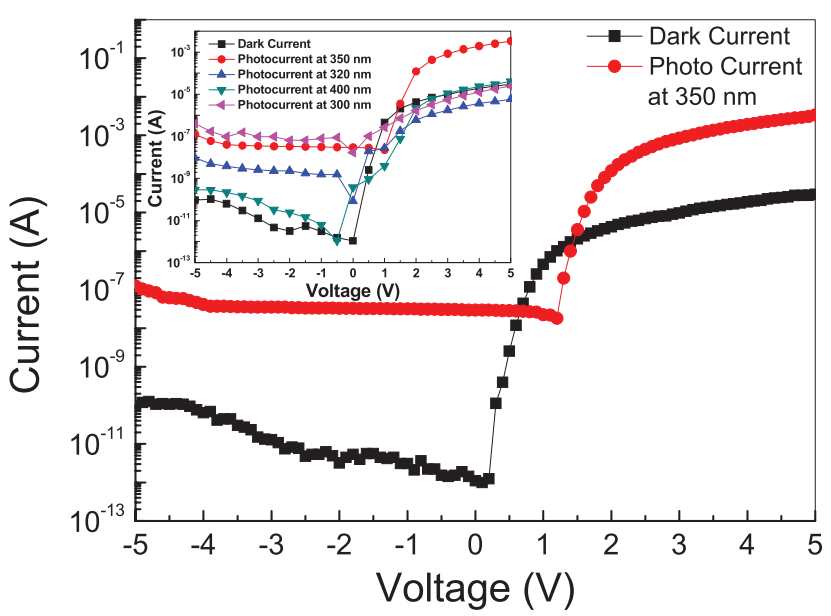

Figure 3. Typical room-temperature $I-V$ characteristics of the graphene/AlGaN/GaN Schottky photodector under dark and illuminated conditions (Inset shows photocurrent above and below $350 \mathrm{~nm})$.

mode corresponding to $1346 \mathrm{~cm}^{-1}$ does not appear in the spectrum, which suggests that the transferred graphene contains few defects $[23,24]$. Moreover, the presence of $G^{*}$ band at $2435 \mathrm{~nm}$ indicating defects exist in the transferred graphene.

The typical room-temperature $I-V$ characteristics of the graphene/AlGaN/GaN Schottky PD under dark and illuminated conditions at $350 \mathrm{~nm}$ are depicted in figure 3 . The fabricated PD shows non-linear behaviour, suggesting that the Schottky barrier is established between the Au electrode/ monolayer graphene and AlGaN/GaN.

The dark current exponentially increases with an increase in the reverse bias, and reaches $9.4 \times 10^{-11} \mathrm{~A}$ at a bias voltage of $-5 \mathrm{~V}$. It is clearly seen from the figure that the dark current is slightly enhanced above $-3 \mathrm{~V}$ reverse bias and is found to be increased by 1 to 2 orders of magnitude, which may be considered as a soft breakdown. However, the photocurrent under $350 \mathrm{~nm}$ monochromatic illumination with UV light is observed to be relatively constant up to the bias voltage of $-4 \mathrm{~V}$ and the photocurrent value is found to be around $3.51 \times 10^{-8} \mathrm{~A}$. The photocurrent measured below and above $350 \mathrm{~nm}$ is found to decrease suggesting that less number of photo carriers is generated and even at $400 \mathrm{~nm}$, photocurrent is very close to dark current as is shown in the inset. However, at $300 \mathrm{~nm}$, photocurrent is marginally increased, there might be more light is absorbed on both $\mathrm{AlGaN}$ and GaN layers and produced large number of photo generated carriers.

The turn-on voltage of the fabricated device was about 1.7 V. The photo-to-dark current contrast ratio was found to be more than three orders of magnitude.

The ideality factor and barrier height, which represent characteristic parameters of the Schottky PD, were extracted from the forward $I-V$ characteristics by fitting the $\ln (I)$ versus $V$ curves using thermionic emission theory. Figure 4 represents the $\ln (I)$ versus $V$ curve of the graphene/AlGaN/GaN Schottky PD.

It is not feasible to obtain an adequate description from the forward $I-V$ curve using a single-diode model. Therefore, an equivalent circuit of two Schottky diodes connected

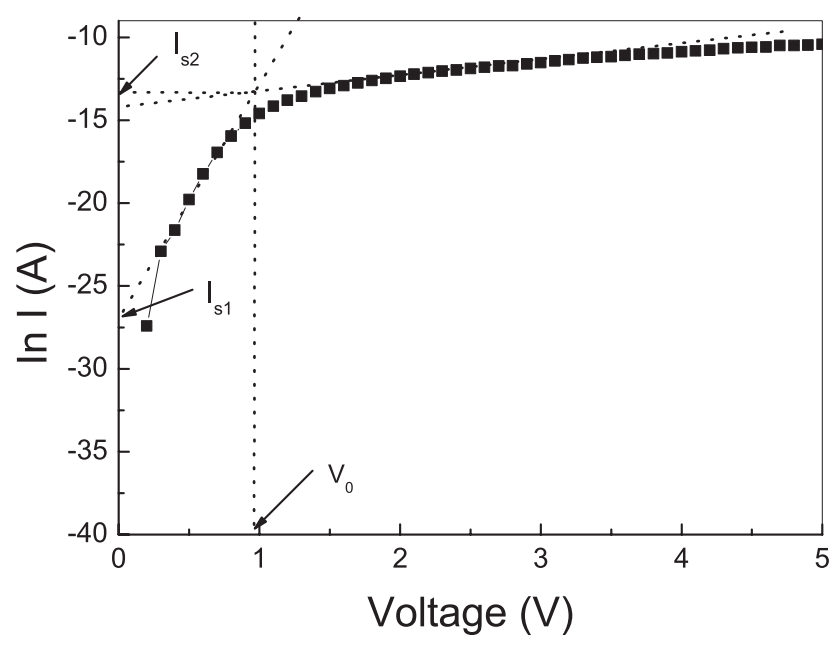

Figure 4. Fitted forward bias $\ln (I)$ versus $V$ curve of the graphene/ AlGaN/GaN Schottky photodector.

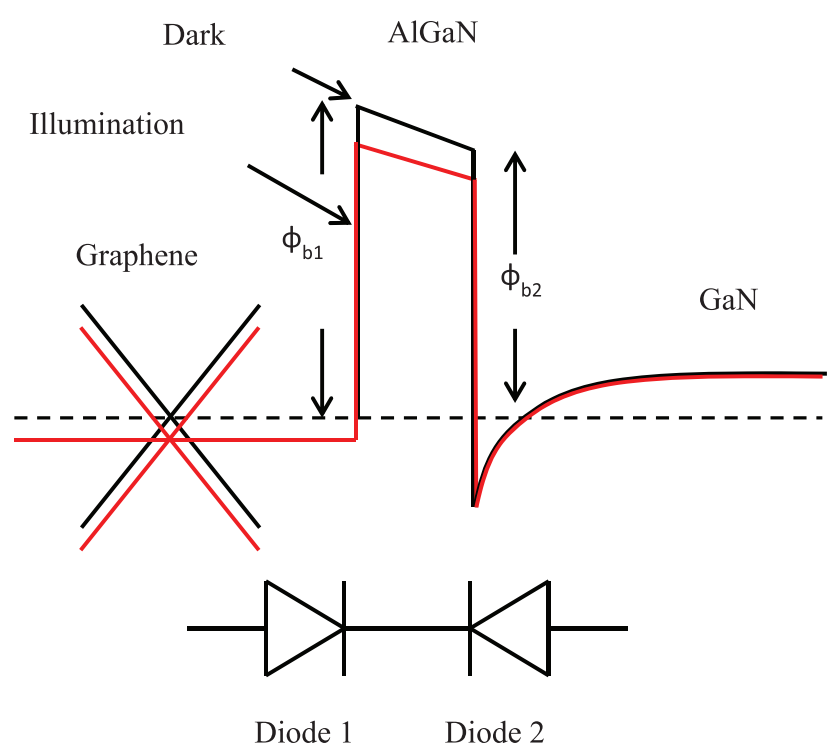

Figure 5. The possible energy band diagram of the graphene/ $\mathrm{AlGaN} / \mathrm{GaN}$ Schottky photodetector modelled as two diodes connected back-to-back in a series equivalent circuit.

back-to-back in series was used, as schematically demonstrated in figure 5; diode 1 represents the Schottky contact between $\mathrm{Au}$ /graphene and the AlGaN barrier layer, whereas diode 2 models the heterojunction of $\mathrm{AlGaN}$ and $\mathrm{GaN}$ [25].

According to the thermionic emission model, the reverse saturation current of the two diodes can be written as follows:

$$
I=I_{s 1}\left[\exp \left(\frac{q V_{1}}{n_{1} k T}\right)-1\right],
$$

where $V_{1}$ is the voltage across diode $1, n_{1}$ is the ideality factor of diode $1, k$ is the Boltzmann constant, and $T$ is the temperature. $I_{s 1}$ can then be expressed as follows:

$$
I_{s 1}=A A^{*} T^{2} \exp \left(-\frac{q \phi_{\mathrm{b} 1}}{k T}\right),
$$

where $q$ is the electron charge, $A$ is the effective Schottky contact area, $A^{*}$ is the Richardson constant $\left(35.8 \times 10^{5}\right.$ 
$\left.\mathrm{A} \cdot \mathrm{cm}^{-2} \cdot \mathrm{K}^{-2}[26]\right)$, and $\phi_{\mathrm{b} 1}$ is the effective barrier height of diode 1 . The current through the reverse-biased diode 2 can then be expressed as:

$$
I=I_{s 2}\left[\exp \left(\frac{q V_{2}}{n_{2} k T}\right)-1\right],
$$

where $V_{2}$ is the applied voltage across diode 2. $I_{s 2}$ can then be expressed as follows:

$$
I_{s 2}=A A^{*} T^{2} \exp \left(-\frac{q \phi_{\mathrm{b} 2} V_{2}}{k T}\right)
$$

where $\phi_{\mathrm{b} 2}$ is the barrier height of diode 2. By applying a Taylor series first-order correction, the above equation can be expressed as:

$$
I=I_{s 2}\left[\exp \left(\frac{q\left|V_{2}\right|}{n_{2} k T}\right)\right]
$$

where the following equations hold:

$$
\begin{gathered}
n_{2}=\frac{1}{\left|\frac{\partial \Phi_{\mathrm{B} 2}}{\partial V_{2}}\right|}, \\
I_{s 2}=A A^{*} T^{2} \exp \left(-\frac{q \phi_{\mathrm{b} 2}(0)}{k T}\right) .
\end{gathered}
$$

Here, $\phi_{\mathrm{b} 2}(0)$ is the barrier height of diode 2 at zero bias, and $n_{2}$ represents the effective ideality factor of diode 2 . The Chen model [25] suggests that the effective ideality factor $n_{2}$ indicates the degree of barrier height $\phi_{\mathrm{b} 2}$ change due to the change of voltage across it. A larger value of $n_{2}$ denotes a smaller change of barrier height. By using these equations, the parameters $I_{s 1}, I_{s 2}, n_{1}$, and $n_{2}$ can be obtained from the forward $I-V$ measurements by linearly fitting the $\ln (I)$ versus $V$ curve in figure 4. More specifically, the parameters $I_{s 1}$ and $n_{1}$ can be obtained by linearly fitting the $\ln (I)$ versus $V$ curve at a low voltage. The slope provides the information of $n_{1}$, whereas the intercept of the fitted straight line yields $I_{s 1}$. On the other hand, the parameters $I_{s 2}$ and $n_{2}$ can be obtained from the linear fit of the $\ln (I)$ versus $V$ curve at an intermediate voltage regime. The product of $A$ and $A^{*}$ in Equations (2) and (7) is not precisely known because the experimental values of the Richardson constant are typically lower than the ideal value, and the effective contact area can be subjected to small variation from point to point. Therefore, in order to determine the values of $\phi_{\mathrm{b} 1}$ and $\phi_{\mathrm{b} 2}(0)$ from the fitting values of $\ln \left(I_{s 1}\right)$ and $\ln \left(I_{s 2}\right)$ without using $A A^{*}$, the subsequent procedure was adopted.

First, the built-in potential across the AlGaN barrier layer at zero bias can be obtained from the following equation [27]:

$$
\left(\frac{\left(\ln I_{s 2}-\ln I_{s 1}\right)}{V_{0}}\right)=\frac{q}{\left(K T n_{1}\right)}=\phi_{\mathrm{b} 1}-\phi_{\mathrm{b} 2}(0) .
$$

The first part of the equation derives from the difference of equations (1) and (7), and $I_{s 1}$ and $I_{s 2}$ are directly obtained from the two linear fits. The obtained values of $I_{s 1}, I_{s 2}, n_{1}$, and $n_{2}$ are $3.04 \times 10^{-16} \mathrm{~A}, 1.63 \times 10^{-6} \mathrm{~A}, 1.57$, and 31.32 , respectively. It should be noted that $V(0)$ is associated with the difference between the positive surface polarization charge $+\sigma$ and the density $N_{\mathrm{D}}$ of the AlGaN/GaN 2DEG at zero bias:

$$
V(0)=q \frac{\sigma-N_{\mathrm{D}}}{C_{\mathrm{AlGaN}}}
$$

where $C_{\mathrm{AlGaN}}=\frac{\varepsilon_{0} \varepsilon_{\mathrm{AlGaN}}}{d}$ is the barrier layer capacitance per unit area, $d$ is the barrier layer thickness $(30 \mathrm{~nm})$, and the relative dielectric permittivity for $\mathrm{Al}_{x} \mathrm{Ga}_{1-x} \mathrm{~N}$ with an $\mathrm{Al}$ concentration of $25 \%$ is determined by using the equation $\varepsilon(x)=-0.5 x+9.5$, which yields $\varepsilon_{\mathrm{AlGaN}}=9.375$. The value of $N_{\mathrm{D}}$ can be obtained by calculating the value of $V(0)$ from the fitting results of equation (8) and the $\sigma$ value from the $C-V$ analysis $\left(\sigma=7.86 \times 10^{11} \mathrm{~cm}^{-2}\right)$.

At this stage, the Fermi energy can be expressed as follows [28]:

$$
E_{\mathrm{F}}(0)=E_{0}(x)+\frac{\pi \hbar^{2}}{m^{*}(x)} n_{S}(x),
$$

where the ground sub-band level of the 2DEG is given by:

$$
E_{0}(x)=\left\{\frac{9 \pi \hbar e^{2}}{8 \varepsilon_{0} \sqrt{m^{*}(x)}} \frac{n_{S}(x)}{\varepsilon(x)}\right\}^{\frac{2}{3}} .
$$

The Fermi level position with respect to the GaN conduction band minimum $\left(E_{\mathrm{Cmin}}\right)$ at zero bias can be expressed as a function of $N_{\mathrm{D}}$ [29]:

$E_{\mathrm{F}}(0)-E_{\mathrm{C} \min }=\frac{\pi \hbar^{2}}{q m_{\mathrm{eff}}} N_{\mathrm{D}}+\frac{1}{q}\left\{\frac{9 \pi \hbar q^{2}}{8 \varepsilon_{0} \varepsilon_{\mathrm{AlGaN}} \sqrt{8 m_{\mathrm{eff}}}}\right\}^{\frac{2}{3}} n_{\mathrm{D}}^{\frac{2}{3}}$

where $\hbar$ is the reduced Planck's constant and $m_{\mathrm{eff}}$ is the $2 \mathrm{DEG}$ effective mass $\left(m_{\mathrm{eff}}=0.22 m_{\mathrm{e}}\right)$. According to the band structure (illustrated in figure 5), the barrier height of diode 2 is:

$$
\phi_{\mathrm{b} 2}(0)=\Delta E_{c}-\left[E_{\mathrm{F}}(0)-E_{\mathrm{Cmin}}\right],
$$

where $\Delta E_{\mathrm{C}}=0.34 \mathrm{eV}$ is the $\mathrm{Al}_{0.25} \mathrm{Ga}_{0.75} \mathrm{~N} / \mathrm{GaN}$ conduction band offset. The barrier height $\phi_{\mathrm{b} 1}$ of diode 1 can be obtained from equation (8). By applying the above procedures, the barrier heights $\phi_{\mathrm{b} 1}$ and $\phi_{\mathrm{b} 2}$ can therefore obtained as 1.14 and $0.228 \mathrm{eV}$, respectively.

The possible energy band diagram of the graphene/ $\mathrm{AlGaN} / \mathrm{GaN}$ Schottky PD is depicted in figure 5. Because of the contact potential that results from the difference of the work function of the two materials, electrons accumulate on the graphene side, and band bending in AlGaN/GaN forms the Schottky barrier in under dark conditions. The electron affinity for $\mathrm{Al}_{0.25} \mathrm{Ga}_{0.75} \mathrm{~N}$ is about $2.7 \mathrm{eV}$ [30], and the work function of the graphene sheet is known to be $4.5 \mathrm{eV}$ below the vacuum level [31]. Thus, when $\mathrm{AlGaN}$ is in contact with the graphene, it is energetically favourable for the photogenerated electrons to be transferred from the conduction band of $\mathrm{AlGaN}$ to the graphene. The graphene side is expected to receive a smaller accumulation of electrons owing to its high carrier mobility. On the other hand, under illumination conditions, AlGaN/GaN absorbs the UV incident light, and graphene serves as a transparent carrier collector. The incident UV light energy (which is larger than the energy gap of 


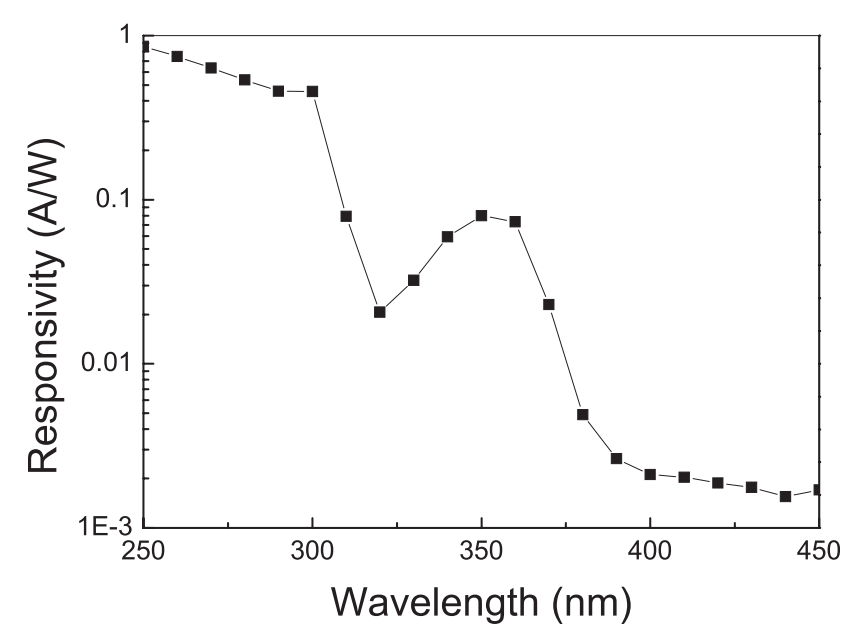

Figure 6. Spectral responsivity of the graphene/AlGaN/GaN Schottky photoditector.

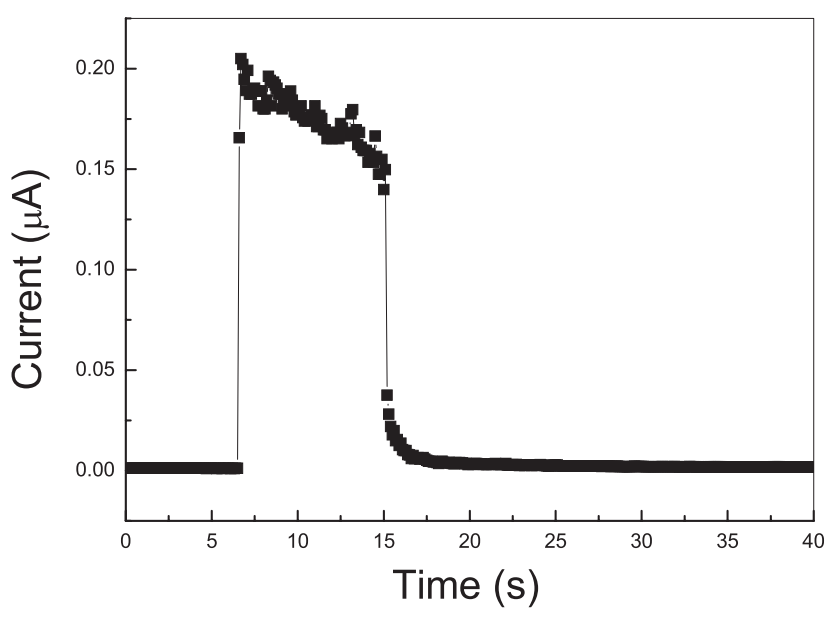

Figure 7. Time response of the graphene/AlGaN/GaN Schottky photoditector.

$\mathrm{AlGaN} / \mathrm{GaN}$ ) is absorbed, and thus electron-hole pairs are generated and rapidly separated by the built-in electric field at the graphene/AlGaN interface.

It should be noted that some impurities may originate from the chemicals used to release the graphene sheet from the $\mathrm{Cu}$ foil, which may exist at the graphene/AlGaN interface. Moreover, desorption of $\mathrm{H}_{2} \mathrm{O}$ molecules can be induced on graphene during illumination, resulting in trapped electrons that may be released to the graphene. The work function of graphene is reduced under UV illumination, which will lower the Schottky barrier height and increase the Fermi level $[32,33]$. The separated electrons can then easily move to the quantum well at the $\mathrm{AlGaN} / \mathrm{GaN}$ interface. It should be noted that because of the excellent transport properties of graphene, the fabricated graphene/AlGaN/GaN-based Schottky UV PD should have larger incident light absorption efficiency than the metal-based AlGaN/GaN Schottky UV PD. This merit makes the graphene/AlGaN/GaN Schottky UV PD a promising candidate for potential applications in the large-scale integration of $\mathrm{GaN}$ technologies.

The responsivity of the graphene/AlGaN/GaN Schottky $\mathrm{PD}$ is shown in figure 6. A small hump at $300 \mathrm{~nm}$ and a clear peak at $350 \mathrm{~nm}$ appear in the spectrum; these features are associated with the intrinsic transitions above the band edges of $\mathrm{AlGaN}$ and $\mathrm{GaN}$, respectively. The estimated responsivities are obtained as 0.56 and $0.079 \mathrm{~A} \mathrm{~W}^{-1}$, respectively, at a bias of $-2 \mathrm{~V}$. The cut-off wavelength of the graphene/AlGaN/GaN Schottky PD is in good agreement with the band gap energy of $\mathrm{Al}_{0.25} \mathrm{Ga}_{0.75} \mathrm{~N}$ and $\mathrm{GaN}$ [34].

Figure 7 presents the time dependent photocurrent of the graphene/AlGaN/GaN Schottky UV PD illuminated by a $350 \mathrm{~nm}$ with a power density of $36 \mu \mathrm{W}$ and chopper frequency of $390 \mathrm{~Hz}$ at a bias voltage of $-2 \mathrm{~V}$. When light is turned on, the PD current increases rapidly from initial value of $1.11 \mathrm{nA}$ to $0.205 \mu \mathrm{A}$ at around $2 \mathrm{~s}$. Once the light is switched off, the photocurrent decreased and returns to a low value at a fast recovery process fist and then slow recovery process. The fast recovery time is estimated about $0.1 \mathrm{~s}$ and slowly recovery time to the initial value within about $13.7 \mathrm{~s}$. The obtained raising and recovery time response of the graphene/AlGaN/ GaN Schottky UV PD is comparable to UV PD based on colloidal $\mathrm{ZnO}$ quantum dot-graphene nanocomposites [35].

\section{Conclusion}

A CVD-grown monolayer graphene-based Schottky PD was successfully fabricated on an AlGaN/GaN template. The $I-V$ characteristics demonstrate a rectifying behaviour, which suggests that the Schottky barrier was indeed achieved at the interface. The graphene/AlGaN/GaN Schottky PD device exhibits low dark current and a large photo-to-dark contrast ratio. Moreover, the graphene/AlGaN/GaN Schottky PD exhibits a responsivities of $0.56 \mathrm{~A} \mathrm{~W}^{-1}$ at $300 \mathrm{~nm}$ and $0.079 \mathrm{~A}$ $\mathrm{W}^{-1}$ at $350 \mathrm{~nm}$ at a bias of $-2 \mathrm{~V}$. The Schottky parameters were evaluated using an equivalent circuit of two diodes connected back-to-back in series. The obtained results demonstrate that graphene can be successfully used as a transparent electrode in AlGaN/GaN-based PDs. Furthermore, the obtained results suggest that the transparent, conductive graphene electrode may be integrated into existing GaN-based technologies.

\section{Acknowledgments}

This research was supported by the Basic Science Research Program (2015R1C1A1A02037326) and the Nano-material Technology Development Program (2009-0082580) through the National Research Foundation of Korea (NRF) funded by the Ministry of Science, ICT, and Future Planning. It was also supported by the 2016 KU Brain Pool of Konkuk University.

\section{References}

[1] Razeghi M and Rogalski A 1996 J. Appl. Phys. 797433

[2] Ozbay E, Biyikli N, Kimukin I, Tut T, Kartaloglu T and Aytur O 2004 IEEE J. Quantum Electron. 10742

[3] Pearton S J, Zolper J C, Shul R J and Ren F 1999 J. Appl. Phys. 861

[4] Kuryatkov V V, Borisov B A, Nikishina S A, Kuchinskii V I, Sokolovskii G S, Song D Y and Holtz M 2006 J. Appl. Phys. 100096104 
[5] Chang K H, Sheu J K, Lee M L, Tu S J, Yang C C, Kuo H S, Yang J H and Lai W C 2010 Appl. Phys. Lett. 97013502

[6] Jiang H and Egawa T 2007 Appl. Phys. Lett. 90121121

[7] Malinowski P E et al 2011 Appl. Phys. Lett. 98141104

[8] Novoselov K S, Geim A K, Morozov S V, Jiang D, Zhang Y, Dubonos S V, Grigorieva I V and Firsov A A 2004 Science 306666

[9] Kim K S et al 2009 Nature 457706

[10] Kim R H et al 2011 Nano Lett. 113881

[11] Tongay S, Lemaitre M, Miao X, Gila B, Appleton B R and Hebard A F 2012 Phys. Rev. X 2011002

[12] Shivaraman S, Herman L H, Rana F, Park J and Spencer M G 2012 Appl. Phys. Lett. 10018311

[13] Tongay S, Lemaitre M, Schumann T, Berke K, Appleton B R, Gila B and Hebard A F 2011 Appl. Phys. Lett. 99102102

[14] An Y, Behnam A, Pop E and Ural A 2013 Appl. Phys. Lett. 102013110

[15] Miao X, Tongay S, Petterson M K, Berke K, Rinzler A G, Appleton B R and Hebard A F 2012 Nano Lett. 122745

[16] Shao D, Yu M, Sun H, Hu T, lian J and Sawyera S 2013 Nanoscale 53664

[17] Ye Y et al 2011 J. Mater. Chem. 2111760

[18] Yi J, Lee J M and Park W I 2011 Sensors Actuators B 155264

[19] Babichev A V, Zhang H, Lavenus P, Julien F H, Egorov A Y, Lin Y T, Tu L W and Tchernycheva M 2013 Appl. Phys. Lett. 103201103

[20] Xu Q, Cheng Q, Zhong J, Cai W, Zhang Z, Wu Z and Zhang F 2014 Nanotechnology 25055501
[21] Fu X W, Liao Z M, Zhou Y B, Wu H C, Bie Y Q, Xu J and Yu D P 2012 Appl. Phys. Lett. 100223114

[22] Kaniyoor A and Ramaprabhu S 2009 Phys. Rep. 47351

[23] Lin F, Chen S W, Meng J, Tse G, Fu X W, Xu F J, Shen B, Liao Z M and Yu D P 2014 Appl. Phys. Lett. 105073103

[24] Kumar M, Noh Y, Polat K, Okyay A K and Lee D 2015 Solid State Commun. 22437

[25] Chen C H, Baier S M, Arch D K and Shur M S 1988 IEEE Trans. Electron Devices 35670

[26] Lv Y, Lin Z, Corrigan T D, Zhao J, Cao Z, Meng L, Luan C, Wang Z and Chen H 2011 J. Appl. Phys. 109074512

[27] Fisichella G, Greco G, Roccaforte F and Giannazzo F 2014 Nanoscale 68971

[28] Shur M S 1998 Mater. Res. Soc. Symp. Proc. 48315

[29] Ambacher O et al 1999 J. Appl. Phys. 853222

[30] Arehart A R, Allerman A A and Ringel S A 2011 J. Appl. Phys. 109114506

[31] Nourbakhsh A, Cahtoro M, Hadipour A, Voseh T, Veen M H V D, Heyns M M, Sels B F and Gendt S D 2010 Appl. Phys. Lett. 97163101

[32] Park P S, Reddy K M, Nath D N, Yang Z, Padture N P and Rajan S 2013 Appl. Phys. Lett. 102153501

[33] Das A et al 2008 Nat. Nanotechnol. 3210

[34] Kumar M, Lee C Y, Sekiguchi H, Okada H and Wakahara A 2013 Semicond. Sci. Technol. 28094005

[35] Son D I, Yang H Y, Kim T W and Park W I 2013 Appl. Phys. Lett. 102021105 